\title{
Transmission and Persistence of Salmonella enterica in Nutrient Solution of Hydroponic Greenhouse Grown Tomatoes
}

\author{
Shannon M. Coleman \\ Department of Food Science and Human Nutrition, Iowa State University, \\ 536 Farmhouse Lane, Ames, IA 50011
}

Bledar Bisha

Department of Animal Science, University of Wyoming, 1000 E. University Avenue, Laramie, WY 82071

\author{
Steven E. Newman ${ }^{1}$ \\ Department of Horticulture and Landscape Architecture, Colorado State \\ University, $301 \mathrm{~W}$. University Avenue, Fort Collins, CO 80523
}

\author{
Marisa Bunning \\ Department Food Science and Human Nutrition, Colorado State University, \\ 234 Gifford Building, Fort Collins, CO 80523
}

Lawrence D. Goodridge

Department of Food Science and Agricultural Chemistry, Food Safety and Quality Program, McGill University, 21,111 Lakeshore Dr., Ste Anne de Bellevue, Quebec, H9X 3V9, Canada

Additional index words. food safety, nutrient film technique, greenhouse tomato

\begin{abstract}
Hydroponic greenhouse tomato production's popularity has grown in the United States to meet the demands for year-round availability of fresh tomatoes. Although Salmonella has been the cause of several foodborne illness outbreaks linked to tomatoes, the potential for contamination in hydroponic production is not well understood. The objectives of this study were to determine whether biweekly inoculation of Salmonella in a hydroponic tomato nutrient solution would lead to Salmonella survival and contamination of the tomato fruit and plants, hydroponic nutrient film technique (NFT) troughs, and water receptacles. An avirulent strain of Salmonella Typhimurium was used to contaminate the nutrient solution at a concentration of $10^{5}$ colony-forming units $(\mathrm{CFU}) / \mathrm{mL}$. Inoculation was conducted on day 0 and biweekly until the termination of project at 12 weeks; samples were filtered and plated on selective media. Leaves and biofilm coupons were collected on day 0 and every 2 weeks postinoculation. Leaf samples were analyzed using culture methods. The biofilm coupons were analyzed using tape fluorescence in situ hybridization (FISH) method. Fruit samples were collected 6 weeks postinoculation until termination of project and analyzed using culture methods. Typical Salmonella morphology of colonies on plates streaked from overnight cultures from plant samples were confirmed by automated ribotyping. A 2 - $\log _{10}$ reduction of cells was observed in water samples 2 days post initial inoculation. Reduction continued over the 2 -week period with few cells surviving until the next inoculation. $S$. Typhimurium was observed on the surface of the root systems. However, a splash incident resulted in low-level contamination of selected leaves and fruit samples. The results of the study indicate that although contaminated hydroponic nutrient solution led to surface contamination of roots, such an event may not pose a high risk of contamination of hydroponically grown fruit.
\end{abstract}

Received for publication 20 July 2016. Accepted for publication 2 Dec. 2016.

We thank the Colorado State University (CSU) Plant Environmental Research Center (PERC) Horticulture Team: Kelly Blume, Vanessa Sandoval, Nicholas Drury; and the CSU Food Safety Team: Alma Perez-Mendez, Jeffrey C. Chandler. This research was funded by the United States Department of Agriculture (USDA) National Institute of Food and Agriculture (NIFA) Grant No. 2009-51110-06067.

${ }^{1}$ Corresponding author. E-mail: scoleman@iastate.edu.
Hydroponic greenhouse production has increased in recent years to help meet the demand for year-round fresh produce availability. The Economic Research Service (ERS) of the United States Department of Agriculture (USDA) has also reported a rise in imports of fresh fruits and vegetables with the average U.S. annual consumption of commercially grown fresh fruits almost $6 \mathrm{~kg}$ higher and fresh vegetables $22.7 \mathrm{~kg}$ higher compared with 2003-05 (Huang and Huang, 2007). Along with consumption increases, foodborne illnesses associated with fresh produce have also increased with an estimated $46 \%$ of foodborne illness outbreaks from 1998 to 2008 attributed to the consumption of produce (Painter et al., 2013). Fresh produce is often consumed raw, bypassing processing steps that could help eliminate pathogens in the event of contamination (Francis et al., 1999). Salmonella enterica and multiple serotypes have been linked with multistate foodborne illness outbreaks attributed to tomatoes, cucumbers, cantaloupe, sprouts, and peppers (Bennett et al., 2015). Tomatoes have been ranked second in fresh produce commodities associated with foodborne pathogens, and these outbreaks have often been linked to $S$. enterica (Anderson et al., 2011; Bennett et al., 2015; Cummings et al., 2001; Fatica and Schneider, 2011; Greene et al., 2008). More than three dozen Salmonella outbreaks were associated with raw tomatoes from 1998 to 2014 (Bennett et al., 2015; CDC, 2015). Tomatoes were linked to a large outbreak of Salmonella in 2008, which resulted in 1442 confirmed illnesses and a $\$ 100$ million dollar loss to the tomato industry (Mody et al., 2011). Tomato-associated outbreaks prompted the U.S. Food and Drug Administration (FDA) to create a "Tomato Team" in collaboration with industry stakeholders, with a goal of examining risk factors associated with tomatoes specifying emphasis on enteric pathogens such as Salmonella and developing intervention steps (United Fresh Produce Association, 2010; US FDA, 1998).

The Produce Safety Rule implemented in FDA's Food Safety Modernization Act (FSMA) sets standards for vegetable and fruit production (US FDA, 2014) in an effort to prevent microbial contamination and reduce foodborne illnesses associated with fresh produce. Fresh produce safety research is top priority to better understand microbial contamination by examining ecological niches of the pathogens and various sources of contamination, which could lead to better intervention steps to control microbial growth (Beuchat, 2002). Contamination of produce with foodborne pathogens may occur through numerous pre- and postharvest handling practices, but one of the most commonly recognized sources of contamination of fresh produce is water: surface, wash, and irrigation (Steele and Odumeru, 2004). Water has been highlighted as a potential source of preharvest contamination of tomatoes in previous studies and reports (Bennett et al., 2015; Lopez-Galvez et al., 2014). Contaminated irrigation water can be a risk with field-grown produce, and it is also a concern during greenhouse production, which typically involves soilless media. The risk associated with foodborne pathogens in irrigation water and nutrient solution is a major issue because of the rapidly growing tomato greenhouse industry (Lopez-Galvez et al., 2014).

Research to improve produce safety has been conducted on laboratory- and fieldgrown tomatoes. Although Salmonella has been the cause of several foodborne illness 
outbreaks linked to tomatoes, the potential for contamination in hydroponic commercial production is not well understood, and little research has focused on the commercial hydroponic setting (Guo et al., 2002; Hintz et al., 2010; Lopez-Galvez et al., 2014; Orozoco et al., 2008). Lower preharvest risk factors are generally associated with hydroponic systems, which are considered to be safer microbially than open-field production (Lopez-Galvez et al., 2014; Orozoco et al., 2008). Miles et al. (2009) examined contamination of tomato plants after a series of alternate watering regimes via irrigation water that contained Salmonella Montevideo. They reported five positive root samples and that Salmonella was able to survive in the fertilizer; however, the tomato fruit was not contaminated with Salmonella. Given the evidence of issues associated with Salmonella and water, more research is needed to examine contamination by Salmonella spp. via greenhouse hydroponic commercial tomato production. The objectives of this study were to determine whether biweekly inoculation of Salmonella in a hydroponic tomato system nutrient solution would lead to survival and contamination of the tomato plants, hydroponic NFT troughs, and water receptacles.

\section{Materials and Methods}

Experimental design. The tomato cultivar Jet Star (Lycopersicon esculentum Mill., 'Jet Star' F1; Harris seeds, Rochester, NY) was selected for all experiments because of its indeterminate growth habit, and heavy vine producing large fruit with resistance to cracking and scarring for internalization analysis. The plants were grown in the Plant Environmental Research Center (PERC) greenhouses on the campus of Colorado State University (Fort Collins, CO). Temperature and relative humidity of the greenhouse facility were monitored using a Wadsworth Control MicroSTEP (Wadsworth Control Systems, Arvada, CO). Deionized water was used for the nutrient solution. Six independent NFT hydroponic systems were constructed with eight plants per system. The NFT systems were divided into two treatment groups including a control group (nutrient solution contained deionized water only) and an experimental group [nutrient solution contained deionized water inoculated with Salmonella enterica subsp. enterica serovar Typhimurium (LT2) biweekly].

Tomato plant growing conditions. Seeds were sown into rockwool gro-blocks $(15 \times 15 \times$ $14 \mathrm{~cm}$ cubes; Grodan BV, The Netherlands) on trays in a greenhouse propagation room. After 6 weeks, the tomato seedlings were trimmed and transplanted where roots were allowed to grow throughout the net pots (Hydrofarms, Santa Fe Springs, CA). The seedlings were staked for support using garden stakes (Bond Manufacturing, Antioch, CA).

Hydroponic system parameters. Net pots were distributed throughout a NFT hydroponic system (eight tomato plants per system). The NFT system was selected because of its ability to be a closed environment and reduce biosafety concerns. Each system was composed of a 1040-L intermediate bulk container (IBC) tote serving as the nutrient reservoir, 95-L recirculating return reservoir (Rubbermaid, Atlanta, GA), and 2-GT50 TSW series NFT hydroponic channels and accessories $(10 \times 5 \mathrm{~cm}$; Grow Tech, Dyersville, IA) contained in an ebb and flood table covered with sheets of black polyethylene (PE) plastic. The nutrient tank was filled to 568 L using a volumetric shutoff valve (Bermadon brand; FarmTek, Dyerville, IA). Each nutrient tank contained an aquarium digital thermometer (Petco, San Diego, CA), air stone (ActiveAqua-Hydrofarm; Petaluma, CA), EC monitor (Milwaukee Instruments, Rocky Mount, NC), and $\mathrm{pH}$ probe monitor (Milwaukee Instruments) sealed in place for daily tank measurements. The nutrient reservoirs were painted with black spray paint to reduce the growth of algae. The NFT system consisted of recirculating system in which a waterfall pump (Flotec, Delavan, WI) located in the nutrient reservoir pumped nutrients through the hydroponic channel where the runoff was collected in the return tank and nutrients pumped back to the nutrient reservoir using a water-removal utility pump with on and off intelligence (Flotec). Plants were transplanted into the NFT system and then tied to a bar suspended from the greenhouse trusses above each system using string and zip ties. The systems were slanted at a $2 \%$ grade for gravity flow.

Maintenance of nutrients and hydroponic system. Nutrients used in the nutrient solution were $0.6 \mathrm{~g} \cdot \mathrm{L}^{-1} \quad 4 \mathrm{~N}-7.8 \mathrm{P}-31.5 \mathrm{~K} \quad(4-18-38$ Chem-Gro Tomato Formula; Hydro-Garden Inc., Colorado Springs, CO), $0.3 \mathrm{~g} \cdot \mathrm{L}^{-1}$ calcium nitrate (Norsk Hydro Agri North America, Inc., Tampa, FL), and $0.3 \mathrm{~g} \cdot \mathrm{L}^{-1}$ magnesium sulfate (Hydro-Garden Inc.). The nutrients levels were maintained at an electrical conductance level of $1.0 \mathrm{dS} \cdot \mathrm{m}^{-1}$. The target $\mathrm{pH}$ was 6.0 and adjusted with $\mathrm{pH}$ Up and $\mathrm{pH}$ Down concentrates [pH Up (potassium hydroxide) and $\mathrm{pH}$ Down (phosphoric acid); Advanced Nutrients, Abbotsford, BC, Canada], which were monitored and recorded daily. Oxygen was incorporated into the system using an air pump with air stones and diffuser. The temperature was monitored using an aquarium digital thermometer and recorded daily. Pollination was performed with daily maintenance of the plants by shaking the flower cluster. No pesticides were used during this experiment.

Bacterial culture and antibiotic susceptibility. An avirulent strain of Salmonella enterica subsp. enterica serovar Typhimurium LT2 was obtained from Dr. Michelle Danyluk [Citrus Research and Education Center (CREC) of the University of Florida, Lake Alfred, FL]. The strain was stored at $-80{ }^{\circ} \mathrm{C}$ in tryptic soy broth (TSB; Difco, Becton Dickinson, Sparks, MD) supplemented with $20 \%$ (v/v) glycerol. $S$. Typhimurium LT2 has been used in previous studies as an avirulent surrogate in BSL1 facilities (PuertaGomez et al., 2013).
S. Typhimurium LT2 was tested for antibiotic sensitivity against a panel of 12 antibiotics using the Kirby-Bauer disk diffusion method for zones of inhibitions at CSU Veterinary Diagnostic Laboratory (Fort Collins, CO). The strain was found to be resistant to amikacin, ceftiofur, cephalothin, gentamicin, and streptomycin. The minimum inhibitory concentration (MIC) was used to provide the baseline for supplementation of media with selected antibiotics. Selective media xylose lysine deoxycholate (XLD) agar and buffered peptone water (BPW) used throughout the research were supplemented at $1 / 2$ MIC concentration of gentamicin $(0.5 \mathrm{mcg} / \mathrm{mL})$ and streptomycin (6 $\mathrm{mcg} / \mathrm{mL}$ ) (Sigma-Aldrich, Saint Louis, MO). Presumptively positive $S$. Typhimurium colonies on XLD media presented as black colonies because of the production of hydrogen sulfide $\left(\mathrm{H}_{2} \mathrm{~S}\right)$.

Inoculum preparation and inoculation. For each inoculation occurrence (day 0 and biweekly), the strain ( $S$. Typhimurium LT2) was activated by streaking on tryptic soy agar (TSA; Becton Dickinson) and incubated at $35 \pm 2{ }^{\circ} \mathrm{C}$ for $24 \mathrm{~h}$. One isolated colony was selected and transferred to $10 \mathrm{~mL}$ of TSB (Becton Dickson) followed by incubation with shaking at $35 \pm 2{ }^{\circ} \mathrm{C}$ for $24 \mathrm{~h}$. Ten milliliters of overnight culture was transferred to $300 \mathrm{~mL}$ of TSB and incubated with shaking at $35 \pm 2{ }^{\circ} \mathrm{C}$ for $24 \mathrm{~h}$. A $10-\mathrm{mL}$ aliquot of the overnight inoculum was removed for enumeration on TSA, to confirm that the initial inoculum concentration was $\approx 9 \log _{10} \mathrm{CFU} / \mathrm{mL}$.

Of the six hydroponic systems, four were inoculated with $S$. Typhimurium by adding $300 \mathrm{~mL}$ of the overnight culture to the $568-\mathrm{L}$ nutrient solution, which resulted in a final inoculum of $5 \log _{10} \mathrm{CFU} / \mathrm{mL}$. The nutrient solution was inoculated on the first day of the study and then every 2 weeks for 12 weeks. The remaining two hydroponic systems served as uninoculated controls with nutrient solution and deionized water only.

Microbial analysis of the nutrient solution. Nutrient solution sample collection occurred every other day for a total of 12 weeks $(n=43)$. Nutrient solution samples $(120 \mathrm{~mL})$ were extracted through sterile tubing attached to a side spout from the nutrient tank into a $118-\mathrm{mL}$ sterile specimen cup. The samples were stored on ice and transported to the laboratory within an hour. One hundred milliliters of nutrient solution was serially diluted in lambda buffer [ $100 \mathrm{~mL} \mathrm{NaCl}, 8 \mathrm{~mm}$ $\mathrm{MgSO} 4 \cdot 7 \mathrm{H}_{2} \mathrm{O}, 50 \mathrm{~mL}$ Tris- $\left.\mathrm{HCl}(\mathrm{pH} 7.5)\right]$ and filtered through a Microcheck beverage filter (Pall ${ }^{\circledR}$ Life Sciences, Port Washington, NY) according to the manufacturer instructions. The filter was placed face up on an XLD agar plate (HiMedia, VWR, Randor, PA) with antibiotic supplements and incubated at $35 \pm$ $2{ }^{\circ} \mathrm{C}$ for $24 \mathrm{~h}$. Colonies were enumerated to determine concentration in CFU/mL. Filtration and direct plating were used to enumerate $S$. Typhimurium from the nutrient solutions.

Sample collection tomato plants. All collected tomato plant portions were analyzed 
for the presence of $S$. Typhimurium. Collection of randomly selected leaf portions of the tomato plants occurred on day 0 and every 2 weeks. Tomato fruit samples were collected 6 weeks post initial inoculation of the hydroponic system using a random selection process. Root samples were collected at the termination of the 12-week project post initial inoculation.

Microbiological analysis of leaf portions. Four mature leaves were randomly selected from the upper and lower portion of the tomato plant (control, $n=32$ per sampling; treated, $n=64$ per sampling; 12 total sample weeks). The four leaves were aseptically removed from the plant using sterilized scissors and transferred into a single sterile stomacher bag (Nasco Whirl-Pak, Fort Atkinson, WI). The leaves from each portion were pooled to create one individual sample, and weights were recorded. A 1:5 ratio (weight to volume) of BPW (Becton Dickson) with antibiotic supplements was added to the sample bag, and the sample was stomached for $90 \mathrm{~s}$. The samples were incubated at $35 \pm 2{ }^{\circ} \mathrm{C}$ for $24 \mathrm{~h}$. The overnight enrichment was streaked onto supplemented XLD agar (HiMedia, VWR). After 24-h incubation at $35 \pm 2{ }^{\circ} \mathrm{C}$, colonies displaying typical Salmonella morphology were considered presumptively positive.

Microbiological analysis of tomato fruit. Green to orange tomato fruits were harvested (to mimic commercial harvesting practices) from the tomato plants (control, $n=182$; treated, $n=180$ ) starting at week 6 post initial inoculation occurrence until the termination of the project at week 12. Tomato fruits were aseptically removed from the plant using sterilized scissors and transferred into individual sterile sample bags.

The total amount of samples collected in a day was split in half into two batches: quarter and whole fruit. One half of the batch of tomatoes was analyzed using the modified whole soak method (whole) to detect surface contamination, and the other half of the batch was analyzed using the modified quarter and stomach method (quarter) to detect internalization contamination as described previously by Wang et al. (2012). The "whole" method required weighing of the whole tomato fruit and a 1:1 test portion $(\mathrm{w} / \mathrm{v})$ of supplemented BPW before placement in the stomacher bag. The "quartered" methods required cutting of tomato fruit into four quarters before placement in Seward ${ }^{\circledR}$ stomacher 400 circulators. The samples were soaked at $4{ }^{\circ} \mathrm{C}$ for $24 \mathrm{~h}$ and then incubated at $35 \pm 2{ }^{\circ} \mathrm{C}$ for $24 \mathrm{~h}$. The enrichment was streaked onto supplemented XLD agar. After 24-h incubation at $35 \pm 2{ }^{\circ} \mathrm{C}$, colonies displaying typical Salmonella morphology were considered presumptively positive and were selected, subcultured on TSA, and further analyzed.

Root analysis. At the termination of the study, the bottom portion of the tomato plants, including net pots and the root systems, was aseptically removed from the NFT system using scissors (control, $n=8$; treated, $n=32$ ). Each root system was individually placed in a sample bag and weighed. A 1:1 test portion (weight to volume) of BPW with antibiotic supplements was added to the stomacher bag. The root samples were massaged by hand to release compacted root samples. The samples were incubated at $35 \pm 2{ }^{\circ} \mathrm{C}$ for $24 \mathrm{~h}$ and streaked onto XLD agar with antibiotic supplements. After 24-h incubation at $35 \pm 2{ }^{\circ} \mathrm{C}$, colonies displaying typical Salmonella morphology were considered presumptively positive and were selected, subcultured on TSA, and further analyzed.

Confirmation by automated RiboPrinter. Presumptive Salmonella colonies isolated from the leaves, fruit, and root samples were confirmed using automated ribotyping. Ribosomal DNA from each isolate was profiled using the RiboPrinter Microbial Characterization System (Dupont Qualicon, Wilmington, DE) in accordance with manufacturer instructions. Briefly, the methodology included selecting single colonies from overnight TSA plates, suspending in sample buffer, and heat-treating before being placed in the automated RiboPrinter Microbial Characterization System. DNA restriction was then performed with PvuII. In this system, the restricted DNA was separated by gel electrophoresis and subsequently transferred to a nylon membrane for Southern blot analyses using DNA probes complementary to ribosomal sequences, yielding a Riboprint pattern. The Riboprint patterns of the isolates were matched to reference patterns using DuPont RiboExplorer Software (Ver. 2.2.0232.0).

Biofilm analysis. The determination of biofilm production was conducted to assess if Salmonella could form biolfilms in the hydroponic setup. Polyvinyl chloride (PVC) type I gray coupons $(2.5 \mathrm{~cm} \times 2.5 \mathrm{~cm}$; Fort Collins Plastics, Fort Collins, $\mathrm{CO}$ ) were hung from the lid of the recirculating return reservoir with string and weighed down using a stainless steel nut. Surfaces of coupons were analyzed for the presence of Salmonella spp. using the tape FISH methodology as described by a previous study (Bisha and Brehm-Stecher, 2010). The FISH method included in situ hybridization procedures with fluorescent probes targeting rRNA to visualize the presence of Salmonella spp. cells extracted from the surface of the biofilm coupon while preserving the microbial community structure. A Leica DM4500 P LED microscope (Leica Camera, Germany) was used for microscopic analyses. Images were captured using Q-Capture Pro7 software (Q Imaging, Surrey, BC, Canada).

Statistical analysis. Each treatment group (S. Typhimurium-treated and control) contained eight tomato plants (total of 48 plants). Leaves and fruit from each group were sampled at random. The correlation between the plant tissue type (root, leaves, or fruit) and control (uninoculated) regarding the presence of $S$. Typhimurium was evaluated using chi square $\left(\chi^{2}\right)$ analysis. Statistical analysis was completed using R Foundation for Statistical Computing ( $\mathrm{R}$ version 2.15.1; Vienna, Austria).

\section{Results}

Survival of $S$. typhimurium in nutrient solution. There were four treated systems (nutrient solution inoculated at $\log _{10}$ $5 \mathrm{CFU} / \mathrm{mL}$ ) and two control systems (uninoculated). Over the course of the experiment, the desired level of $S$. Typhimurium at $\log _{10} 5 \mathrm{CFU} / \mathrm{mL}$ was obtained in the nutrient solution at every inoculation event as shown in Fig. 1. No $S$. Typhimurium was recovered from the control tanks. There was a $2-\log _{10}$ reduction observed between the initial inoculation event and the next sampling time point $(2 \mathrm{~d}$ post initial inoculation). The reduction continued until the next inoculation period 2 weeks later. This suggested that $S$. Typhimurium did not survive well in the conventional hydroponic nutrient solution.

Tomato plant analysis. There were 32 tomato plants in the treated hydroponic systems and 16 tomato plants in the control (uninoculated) hydroponic systems. Leaf samples were obtained from upper and lower portions of the plant and pooled for analysis on day 0 and biweekly. At the start of week 8 , one of the treated hydroponic system plants broke free from the suspension support, and the hydroponic system was damaged. The

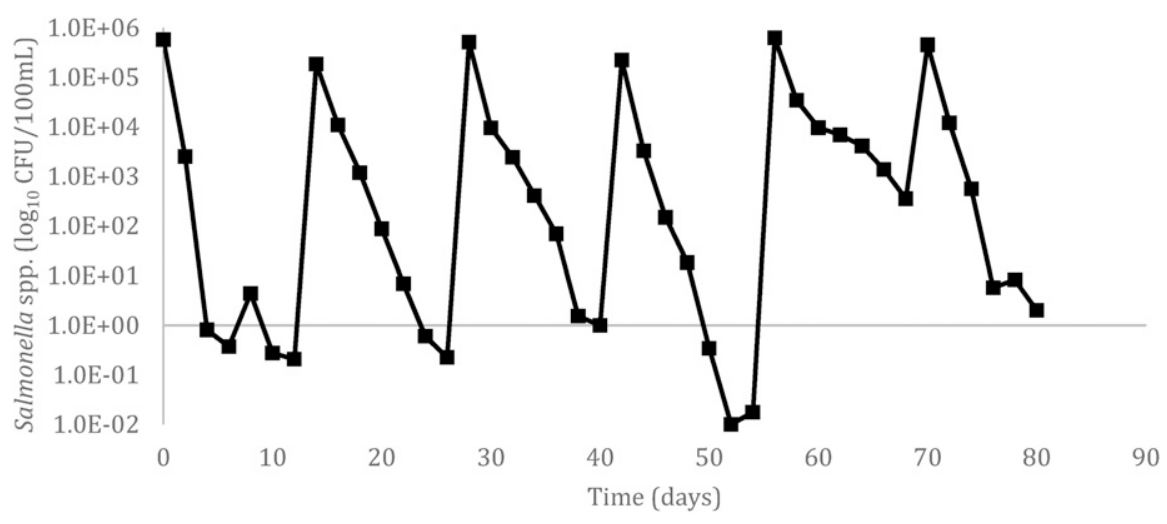

Fig. 1. Changes in avirulent Salmonella enterica serovar Typhimurium LT2 levels $\left(\log _{10} \mathrm{CFU} / 100 \mathrm{~mL}\right)$ in hydroponic nutrient solution during $82 \mathrm{~d}$ (duration of project 12 weeks). $S$. Typhimurium was not recovered from the control nutrient solution tanks and not shown in this figure. 
damaged piping system allowed the nutrient solution to be splashed throughout the system. The piping system was repaired, but the plant samples remained damaged. At week 11, a pooled leaf sample from the lower portion of the damaged tomato plant in the treated hydroponic system confirmed positive for $S$. Typhimurium via automated ribotyping as shown in Table 1 . No $S$. Typhimurium confirmation was observed from any of the other leaf samples taken from both treated and control plants from the other systems.

Tomato fruit samples were collected 6 weeks post initial inoculation and every week until the termination of the project at 12 weeks. One of the quartered tomato fruit samples from the lower portion of a treated plant tested positive for $S$. Typhimurium as shown in Table 1. Both positive leaf and tomato samples had contact with the contaminated nutrient solution from the inoculated tank because of the suspension support collapsing, damaging the system, and causing splashing of inoculated nutrient solution on the plant. No $S$. Typhimurium colonies were observed from any of the other tomato fruit or leaf samples taken from both treated and control plants as shown in Table 1. No $S$. Typhimurium colonies were found on roots from the control plants where $43.5 \%$ of the roots sampled from plants grown in the inoculated systems tested positive for $S$. Typhimurium $(P<0.05)$.

Biofilm analysis. Tape FISH analyses on PVC coupons were performed as described previously by Bisha and Brehm-Stecher (2010). No $S$. Typhimurium cells were observed on the PVC coupons from both treated and control tanks. Coupon surfaces were also enriched in BPW and streaked out onto XLD agar, and typical colonies were not observed.

\section{Discussion}

This study aimed to investigate whether continuous contamination of nutrient solution with $S$. Typhimurium would result in microbial survival and contamination of hydroponically grown tomato plants, hydroponic troughs, and water receptacles. Previous studies have examined risk factors associated with greenhouse or hydroponically grown tomatoes. For example, Guo et al. (2002) observed a Salmonella concentration of more than $3.38 \log _{10} \mathrm{CFU} / \mathrm{g}$

Table 1. Number of positive and negative results from hydroponic tomato plant samples (Lycopersicon esculentum Mill. 'Jet Star' F1) [tomato fruit (quartered and whole), leaves (upper and lower), and roots]. All presumptively positive Salmonella enterica serovar Typhimurium samples confirmed using automated RiboTyping ${ }^{\circledR}$.

\begin{tabular}{lllcc}
\hline Sampling point & Sample type & Control/treated & Number of positive samples & Prevalence (\%) \\
\hline Tomatoes & Quartered & Control & $0 / 85$ & 0 \\
& & Treated & $1 / 79$ & 1.2 \\
& \multirow{2}{*}{ Whole } & Control & $0 / 97$ & 0 \\
& & Treated & $0 / 101$ & 0 \\
Leaves & \multirow{2}{*}{ Upper } & Control & $0 / 112$ & 0 \\
& & Treated & $0 / 224$ & 0 \\
& Lower & Control & $0 / 112$ & 0.4 \\
& & Treated & $1 / 223$ & 0 \\
\multirow{2}{*}{ Root } & Whole & Control & $0 / 16$ & 43.5 \\
& & Treated & $10 / 23^{z}$ & \\
\hline
\end{tabular}

${ }^{\mathrm{z}}$ Significant at $P \leq 0.05$ by $\chi^{2}$ (chi squared) analysis.

in the hypocotyls-cotyledons, stems, and leaves of plants grown for $9 \mathrm{~d}$ with continuous exposure to Salmonella-contaminated Hoagland nutrient solution, regardless of root conditions. Similarly, after continuous contact with Salmonella-contaminated irrigation water, contamination was observed in $65 \%$ of the roots, $40 \%$ of stems, $10 \%$ of leaves, and $6 \%$ of internal fruit samples (Hintz et al., 2010). However, Miles et al. (2009) examined contamination of tomato plants after a series of alternate watering regimes by irrigation water that contained Salmonella Montevideo. Five root samples tested positive, and Salmonella was able to survive in the fertilizer; however, the tomato fruit was not contaminated. In the current study, we observed consistent contamination of roots with $S$. Typhimurium, but it was not found in leaves, or the fruit over the course of the study, with the exception for the instances in which the tomato plants and fruit directly contacted the contaminated nutrient solution. Our results suggest that the nutrient solution, if contaminated with Salmonella, could lead to contamination of hydroponic tomato root system.

Zhou et al. (2014) examined the impact of tomato fruit immersion times in water containing Salmonella and reported that longer immersion time had an effect on internalization, with internalized bacteria persisting for 2 weeks of storage. Other studies have shown that $S$. Typhimurium could be internalized into tomato plants via leaves inoculated with suspension of $10^{9} \mathrm{CFU} / \mathrm{mL}$ Salmonella with surfactant Silwet L-77 without inducing any colonization in the tomato plants ( $\mathrm{Gu}$ et al., 2011). In this study, we observed contamination on a single tomato fruit and pooled leaf sample after one plant collapsed and had contact with the inoculated water, although it was not determined whether Salmonella was present internally. Even if plants and fruits should come in contact with contaminated nutrient solution in a hydroponic operation, subsequent survival and growth of Salmonella will likely depend on the contaminating serovar. For example, Shi et al. (2007) used serovars associated with tomatolinked outbreaks of salmonellosis (Javiana, Montevideo, and Newport) and those typically isolated from animal or clinical infection serovars (Dublin, Enteritidis, Hadar,
Infantis, Typhimurium, and Senftenberg) to test the survival from the inoculated flowers of growing plants. It was discovered that serovar Montevideo is more adapted to survival within tomatoes and was recovered from $90 \%$ of the fruit screened. Both $S$. Enteritidis, Typhimurium and Dublin were less adaptive to grow on ripened intact tomatoes.

The literature contains conflicting results with respect to the fate of tomato plants that are contaminated with $S$. Typhimurium via the roots. In our study, we observed $S$. Typhimurium contamination of root systems $(43 \%)$ through contaminated nutrient solution, which agrees with the finding of earlier studies (Hintz et al., 2010). The researchers also observed that $S$. Newport might be associated with the root system and to a lesser degree with the stem and leaves of tomato plants when introduced to contaminated irrigation water (Hintz et al., 2010). Similarly, Miles et al. (2009) found that the serotype Montevideo survival was observed in five of their root samples, whereas all tomato fruits tested negative. On the other hand, Montevideo did survive in the commercially available fertilizer used in the same study. Nevertheless, there was one instance when inoculation of the roots led to contamination of other parts of the plant especially in the presence of a plant pathogen such as Ralstonia solanacearum (Pollard et al., 2014). Guo et al. (2002) has shown that a hydroponic system with direct root inoculation with a five serotype mixture of $S$. enterica [Montevideo (serogroup $\mathrm{C}_{1}$ ), Michigan (serogroup J), Poona (serogroup $\mathrm{G}$ ), Hartford (serogroup $\mathrm{C}_{1}$ ), and Enteritidis (serogroup D)] could lead to contamination of the entire plant and that Salmonella could survive in or on tomato fruit from the time of inoculation at the flowering stage through fruit development and maturation. Direct inoculation of the roots was the method used to introduce Salmonella in the abovementioned studies, whereas contamination of the roots occurred via an indirect mechanism (contaminated nutrient solution) in our study. Other researchers have found that using this approach can have an alternative effect on the whole environment. For example, plants irrigated with contaminated water have been found to have larger populations of Salmonella than those grown in infected soil (Barak et al., 2011).

Biofilms are defined as a structured community of bacterial cells enclosed in a selfproduced polymeric matrix adhered to a surface (Steenackers et al., 2012). Its formation and the accumulation of microorganisms on surfaces depend on various factors such as composition of surface materials, microbial occurrence in water, concentration, temperature, hydraulics of systems, and concentration and quality of nutrients and disinfectants (Zacheus et al., 2000). Typically, biofilms are common in aquatic environments such as recirculating systems because they can harbor pathogenic organisms through the water and solid interface on tanks and equipment (King et al., 2004; Zacheus et al., 2000). Thus, we were interested to determine 
whether Salmonella could form biofilms in a hydroponic system. During our 12-week study, we did not observe any formation of biofilm on the PVC coupons collected from the recirculating tank. This may have been because $S$. Typhimurium did not survive well in the nutrient solution, and there were not enough bacteria to colonize the PVC coupons. However, other studies have found total counts of heterotrophic bacteria slightly higher on PE than on PVC (Zacheus et al., 2000). Previous studies have shown that Salmonella's flagella play a major role in biofilm formation and the attachment to PVC surfaces (Kim and Wei, 2009; Mireles et al., 2001; Stafford and Hughes, 2007).

Determining the survival of Salmonella spp. in conventional nutrient solution used in a hydroponic tomato operation was one of the major objectives of this study. More specifically, we were interested in assessing if Salmonella contamination of groundwater could lead to nutrient solution contamination and subsequent contamination of the tomato plants. Although groundwater is less likely to be contaminated with food and waterborne pathogens than surface water (Fawell and Nieuwenhuijsen, 2003), there have been outbreaks of waterborne illness associated with contaminated groundwater. This highlights the possibility that groundwater, which is often untreated, could serve as a vehicle by which hydroponically grown tomatoes and other fresh produce could be contaminated with foodborne pathogens such as Salmonella. For example, a major waterborne outbreak of $S$. Typhimurium in Alamosa, CO, in 2008 was linked to contaminated groundwater (Ailes et al., 2013) resulting in 434 illnesses, 20 hospitalizations, and one death. Untreated groundwater is the second most common cause of intestinal illness, which often occurs in nonmunicipal water systems such as camps or outdoor workplaces and one occurred in a meeting facility (Beer et al., 2015).

In conclusion, it was observed in this study that continuous inoculation of $S$. Typhimurium in a hydroponic system nutrient solution was capable of contaminating the root surface of the tomato plants, but did not appear to contaminate the leaves or be internalized to an observable extent in the fruit portion of tomato plants. However, the contamination of roots may raise concerns with other hydroponic crops, such as leafy greens, because of the closer proximity of the vegetative portion to the roots. As hydroponic vegetable production continues to increase, more research is needed to address these food safety issues.

\section{Literature Cited}

Ailes, E., P. Budge, M. Shankar, S. Collier, W. Brinton, A. Cronquist, M. Chen, A. Thornton, M.J. Beach, and J.M. Brunkard. 2013. Economic and health impacts associated with a Salmonella Typhimurium drinking water outbreak-alamosa, CO, 2008. PloS One 8(3): e57439, doi: 10.1371/ journal.pone. 0057439 .

Anderson, M., L.-A. Jaykus, S. Beaulieu, and S. Dennis. 2011. Pathogen-produce pair attribution risk ranking tool to prioritize fresh produce commodity and pathogen combinations for further evaluation (P3ARRT). Food Control 22(12):1865-1872, doi: 10.1016/j.foodcont. 2011.04 .028 .

Barak, J.D., L.C. Kramer, and L.-y. Hao. 2011. Colonization of tomato plants by Salmonella enterica is cultivar dependent, and type 1 trichomes are preferred colonization sites. Appl. Environ. Microbiol. 77(2):498-504, doi: 10.1128/ AEM.01661-10.

Beer, K D, J W Gargano, V A Roberts, and V R Hill. 2015. Surveillance for waterborne disease outbreaks associated with drinking water-United States, 2011-2012. Morb Mortal Weekly Report.

Bennett, S.D., K.W. Littrell, T.A. Hill, M Mahovic, and C. Barton Behravesh. 2015. Multistate foodborne disease outbreaks associated with raw tomatoes, United States, 1990-2010: A recurring public health problem. Epidemiol. Infect. 143(7):1352-1359, doi: $10.1017 / \mathrm{S} 0950268814002167$.

Beuchat, L.R. 2002. Ecological factors influencing survival and growth of human pathogens on raw fruits and vegetables. Microbes Infect. 4 (4):413-423.

Bisha, B. and B.F. Brehm-Stecher. 2010. Combination of adhesive-tape-based sampling and fluorescence in situ hybridization for rapid detection of Salmonella on fresh produce. J. Vis. Expt. 44:E2308, doi: 10.3791/2308.

CDC. 2015. Foodborne Outbreak Online Database (FOOD Tool). 4 Sept. 2014. <http:// wwwn.cdc.gov/foodborneoutbreaks/>.

Cummings, K., E. Barrett, J.C. Mohle-Boetani, J.T Brooks, J. Farrar, T. Hunt, A. Fiore, K. Komatsu, S.B. Werner, and L. Slutsker. 2001. A multistate outbreak of Salmonella enterica serotype baildon associated with domestic raw tomatoes. Emerg. Infect. Dis. 7(6):1046-1048, doi: 10.3201/ eid0706.010625.

Fatica, M.K. and K.R. Schneider. 2011. Salmonella and produce: Survival in the plant environment and implications in food safety. Virulence 2 (6):573-579, doi: 10.4161/viru.2.6.17880.

Fawell, J. and M.J. Nieuwenhuijsen. 2003. Contaminants in drinking water. Br. Med. Bull. 68 (1):199-208, doi: 10.1093/bmb/ldg027.

Francis, G.A., C. Thomas, and D. O'beirne. 1999. The microbiological safety of minimally processed vegetables. Intl. J. Food Sci. Technol. 34(1):1-22, doi: 10.1046/j.1365-2621.1999.00253.x.

Greene, S.K., E.R. Daly, E.A. Talbot, L.J. Demma, S. Holzbauer, N.J. Patel, and T.A. Hill. 2008. Recurrent multistate outbreak of Salmonella newport associated with tomatoes from contaminated fields, 2005. Epidemiol. Infect. 136(2):157-165, doi: $10.1017 / \mathrm{S} 095026880700859 \mathrm{X}$.

Gu, G., J. Hu, M.J. Cevallos-Cevallos, S.M. Richardson, J.A. Bartz, and A.H.C. van Bruggen. 2011. Internal colonization of Salmonella enterica serovar Typhimurium in tomato plants. PloS One 6(11): e27340, doi: 10.1371/ journal.pone.0027340.

Guo, X., M.W. van Iersel, J. Chen, R.E. Brackett, and L.R. Beuchat. 2002. Evidence of association of Salmonellae with tomato plants grown hydroponically in inoculated nutrient solution. Appl. Environ. Microbiol. 68(7):3639-3643, doi: 10.1128/AEM.68.7.3639-3643.2002.

Hintz, L.D., R.R. Boyer, M.A. Ponder, and R.C. Williams. 2010. Recovery of Salmonella enterica newport introduced through irrigation water from tomato (Lycopersicum esculentum) fruit, roots, stems, and leaves. HortScience 45:675-678.

Huang, S. and K. Huang. 2007. Increased U.S. imports of fresh fruit and vegetables. USDA ERS Economic
Research Service. 4 Sept. 2014. <http://www.ers. usda.gov/media/187841/fts32801_1_pdf $>$.

Kim, S.-H. and C.-I. Wei. 2009. Molecular characterization of biofilm formation and attachment of Salmonella enterica serovar typhimurium DT104 on food contact surfaces. J. Food Prot. 72(9):1841-1847.

King, R.K., G.J. Flick, Jr., D. Pierson, S.A. Smith, G.D. Boardman, and C.W. Coale, Jr. 2004. Identification of bacterial pathogens in biofilms of recirculating aquaculture systems. J. Aquat. Food Prod. Technol. 13(1):125-133, doi: 10.1300/ J030v13n01_11.

Lopez-Galvez, F., A. Allende, F. Pedrero-Salcedo, J.J. Alarcon, and M.I. Gil. 2014. Safety assessment of greenhouse hydroponic tomatoes irrigated with reclaimed and surface water. Intl. J. Food Microbiol. 191(17):97-102, doi: 10.1016/ j.ijfoodmicro.2014.09.004.

Miles, J.M., S.S. Sumner, R.R. Boyer, R.C. Williams, J.G. Latimer, and J.M. McKinney. 2009. Internalization of Salmonella enterica serovar montevideo into greenhouse tomato plants through contaminated irrigation water or seed stock. J. Food Prot. 72(4):849-852.

Mireles, J.R., A. Toguchi, and R.M. Harshey. 2001. Salmonella enterica serovar typhimurium swarming mutants with altered biofilm-forming abilities: Surfactin inhibits biofilm formation. J. Bacteriol. 183(20):5848-5854, doi: 10.1128/ JB.183.20.5848-5854.2001.

Mody, R.K., L.G. Sharon A Greene, A. Sever, S. Pichette, I. Zambrana, and T. Dang. 2011. National outbreak of Salmonella serotype saintpaul infections: Importance of Texas restaurant investigations in implicating jalapeño peppers. PloS One 6(2):e16579, doi: 10.1371/journal. pone. 0016579 .

Orozoco, L.R., M.H. Iturriaga, M.L. Tamplin, P.M Frantamico, J.E. Call, J.B. Luchansky, and E.F. Escartin. 2008. Animal and environmental impact on the presence and distribution of Salmonella and Escherichia coli in hydroponic tomato greenhouses. J. Food Prot. 714676-683.

Painter, J.A., R.M. Hoekstra, T. Ayers, R.V. Tauxe, C.R. Braden, F.J. Angulo, and P.M. Griffin. 2013. Attribution of foodborne illnesses, hospitalizations, and deaths to food commodities by using outbreak data, United States, 1998 2008. Emerg. Infect. Dis. 19(3):407-415.

Pollard, S., J.D. Barak, R. Boyer, M. Reiter, G. Gu, and S. Rideout. 2014. Potential interactions between Salmonella enterica and Ralstonia solanacearum in tomato plants. J. Food Prot. 77(2):320-324, doi: 10.4315/0362-028X.JFP-13-209.

Puerta-Gomez, A.F., J. Kim, R.G. Moreira, G.A. Klutke, and M.E. Castell-Perez. 2013. Quantitative assessment of the effectiveness of intervention steps to reduce the risk of contamination of ready-to-eat baby spinach with Salmonella. Food Control 31(2):410-418, doi: 10.1016/ j.foodcont.2012.10.022.

Shi, X., A. Namvar, M. Kostrzynska, R. Hora, and K. Warriner. 2007. Persistence and growth of different Salmonella serovars on preand postharvest tomatoes. J. Food Prot. 70 (12):2725-2731.

Stafford, G.P. and C. Hughes. 2007. Salmonella typhimurium flhE, a conserved flagellar regulon gene required for swimming. Microbiology, 153:541-547, doi: 10.1099/mic.0.2006/ 002576-0.

Steele, M. and J. Odumeru. 2004. Irrigation water as source of foodborne pathogens on fruit and vegetables. J. Food Prot. 67(12):2839-2849.

Steenackers, H., K. Hermans, J. Vanderleyden, and S.C.J. De Keersmaecker. 2012. Salmonella biofilms: An overview on occurrence, structure, 
regulation and eradication. Food Res. Intl. 45(2): 502-531, doi: 10.1016/j.foodres.2011.01.038.

United Fresh Produce Association. 2010. Food safety programs and auditing protocol for the fresh tomato supply chain. doi: $10.1111 /$ j.13652621.1985.tb13322.x/full.

US FDA. 1998. Guide to Minimize Microbial Food Safety Hazards for Fresh Fruits and Vegetables. 4 Sept. 2014. <https://www.fda.gov/ohrms/ dockets/98fr/97n0451.pdf>.

US FDA. 2014. FSMA Facts: Proposed Rule on Sanitary Transportation of Human and Animal
Food. 31 Jan. 2014. <http://www.fda.gov/ downloads/Food/GuidanceRegulation/FSMA/ UCM383764.pdf>.

Wang, H., V.S. Gill, K.A. Irvin, M. Byrd, C.M. Bolger, J. Zheng, E.E. Dickey, R.E. Duvall, A.P. Jacobson, and T.S. Hammack. 2012. Recovery of Salmonella from internally and externally contaminated whole tomatoes using several different sample preparation procedures. J. AOAC Intl. 95(5):1452-1456, doi: 10.5740/ jaoacint.11-463.
Zacheus, O.M., E.K. Iivanainen, T.K. Nissinen, M.J. Lehtola, and P.J. Martikainen. 2000. Bacterial biofilm formation on polyvinyl chloride, polyethylene and stainless steel exposed to ozonated water. Water Res. 34(1):63-70, doi: 10.1016/S0043-1354(99)00113-X.

Zhou, B., Y. Luo, X. Nou, Y. Yang, Y. Wu, and Q. Wang. 2014. Effects of postharvest handling conditions on internalization and growth of Salmonella enterica in tomatoes. J. Food Prot. 77(3):365-370, doi: 10.4315/0362-028X.JFP13-307. 